\title{
The Efficacy and Safety of Subcutaneous Immunotherapy in Mite-Sensitized Subjects With Asthma: A Meta-Analysis
}

\author{
Yanming Lu MD, Lingyun Xu MM, Min Xia MM, Yaqin Li MM, and Lanfang Cao MM
}

\begin{abstract}
BACKGROUND: Subcutaneous immunotherapy (SCIT) is widely used in the management of allergic diseases such as allergic asthma. We aimed to conduct a systematic review and metaanalysis to evaluate the efficacy and safety of SCIT in mite-sensitized subjects with asthma. METHODS: Literature from January 1990 to February 2013 on the efficacy and safety of SCIT for mite-sensitized asthma patients was searched in electronic databases, including the Cochrane Library, MEDLINE, Embase, PubMed, China Knowledge Resource Integrated Database, Wanfang, and Vendor Information Pages. Data were extracted from randomized controlled trials (RCTs) according to the selection criteria by 2 investigators independently. The quality of included trials was evaluated according to the Jadad scale standard. RESULTS: A total of 796 subjects from 19 different RCTs were included in this analysis. SCIT significantly reduced the asthma symptom scores (standardized mean difference of $-0.94,95 \% \mathrm{CI}-1.58$ to $-0.29, P=.004$ ) and the asthma medication scores (standardized mean difference of $-1.06,95 \%$ CI -1.70 to $-0.42, P=.001$ ) compared with the control group. However, there were no significant differences between subjects receiving SCIT and the control group in lung function (peak expiratory flow, percent-of-predicted $\mathrm{FEV}_{1}$, percent-of-predicted FVC) and specific antibody (allergen-specific immunoglobulin E) levels of blood serum $(P>.05)$. In the studies containing data on safety, the incidences of systemic and local adverse reactions were $9.1 \%(8 / 88)$ and $17.2 \%(23 / 134)$, respectively, in subjects treated with SCIT, and no severe adverse events were reported. CONCLUSIONS: Our results suggest that SCIT is helpful in alleviating symptoms and reducing medication used in mite-sensitive asthma subjects, but with no improvement in lung function. The safety of SCIT is acceptable. Key words: asthma; subcutaneous; specific immunotherapy; mite; meta-analysis; efficacy; safety. [Respir Care 2015;60(2):269-278. (C) 2015 Daedalus Enterprises]
\end{abstract}

\section{Introduction}

Allergen-specific immunotherapy (AIT) is currently a key part of the allergic asthma management prevention strategy of the Global Initiative for Asthma (GINA). AIT

\footnotetext{
The authors are affiliated with the Department of Pediatrics, Ren Ji Hospital, School of Medicine, Shanghai Jiao Tong University, Shanghai, China.

The authors have disclosed no conflicts of interest.

Correspondence: Yanming Lu MD, Department of Pediatrics, Ren Ji Hospital, School of Medicine, Shanghai Jiao Tong University, Middle Shandong Road, Shanghai 200001, China. E-mail: luyanming_1980@163.com.
}

DOI: $10.4187 /$ respcare.03399 is the only available treatment method that addresses the causes of asthma and is class A-recommended based on the results of evidence-based medicine. ${ }^{1}$ Presently, AIT is used extensively by physicians around the world. The most current research and application of allergen preparations involve house dust mites, grass pollen, cat dander, dog dander, and other allergens mixed. Subcutaneous immunotherapy (SCIT) and sublingual immunotherapy are the 2 most prescribed routes for administering AIT. SCIT is now widely used in the management of allergic diseases, including allergic asthma. Many doctors are still concerned about the clinical efficacy and safety of SCIT, and they think it is less effective than inhaled corticosteroids and bronchodilator agents. During a 2007 session at the European Academy of Allergy and Clinical Immunology (EAACI) annual congress in Gothenburg, Sweden, Dr Peter Barnes stated that "there is no need to perform 
allergy tests in asthma, since asthma treatment is the same, whether it is an allergic asthma or not." This comment has triggered a debate about the efficacy of SCIT. ${ }^{2}$

Adkinson et $\mathrm{al}^{3}$ conducted a double-blind, placebocontrolled trial of multiple-allergen immunotherapy in 121 allergic children with moderate-to-severe perennial asthma. They tracked medication use and symptoms and found no differences in symptom scores, peak flows, and medication use between the treated and control groups. In contrast, in a multi-center, randomized, double-blind, placebocontrolled clinical study from China, the treated group received a standardized extract of Dermatophagoides pteronyssinus SCIT for $1 \mathrm{y}$. The results showed that the SCIT treatment produced significant improvement in subjects with mild-to-moderate symptoms of allergic asthma and reduced the use of symptomatic medication, and no severe systemic adverse reactions were reported. ${ }^{4}$ The difference between the 2 studies may be the selection of subjects and the different doses of allergen. In recent years, most studies have had limited numbers of subjects and used different allergens without a standardized course of treatment or end point indicators. The quality of studies reported in the literature is not uniform, and some were historical control trials of efficacy. Most AIT studies in China involved desensitization therapy for dust mites. Thus, with the goal of further clarifying the effectiveness and safety of SCIT for dust mite allergic asthma, we evaluated all randomized controlled trials (RCTs) published in a 13-y period using meta-analysis to provide a reference for clinical practice.

\section{Methods}

\section{Inclusion and Exclusion Criteria}

Study Design. RCTs were selected for our study based on the following criteria. Trials were excluded if they were quasi-randomized, regardless of whether they were blinded. Trials were chosen (1) regardless of the gender, age, race, and nationality of subjects and if they included (2) subjects who had either asthma alone or in combination with other symptoms (allergic rhinitis, conjunctivitis, dermatitis); (3) subjects who all demonstrated positive skin prick tests or specific antibodies in serum to house dust mites, storage mites, etc; (4) subjects in whom no reactions to other air-borne allergens besides dust mites were found; (5) subjects with no specific immunotherapy history before the clinical studies were performed; and (6) subjects whose pulmonary function tests showed $\mathrm{FEV}_{1}>70 \%$ of predicted values. Exclusion criteria were: (1) studies that did not include the primary and secondary outcome measures listed in the following paragraph. (2) studies that included duplicate reports (the most complete data were used), (3) non-clinical studies, (4) data could not

\section{QUICK LOOK}

\section{Current knowledge}

Allergen-specific immunotherapy is a key part of the allergic asthma management prevention strategy of the Global Initiative for Asthma. It is the only available treatment that addresses the causes of asthma and is recommended based on the results of evidence-based medicine.

\section{What this paper contributes to our knowledge}

Subcutaneous immunotherapy (SCIT) was helpful in alleviating symptoms and reducing medication usage in mite-sensitive asthma subjects. SCIT was safe, but did not result in any improvement in subjects' lung function.

be merged or were from poor-quality literature sources, (5) clinical studies focused on other diseases, and (6) studies in which $>20 \%$ of subjects were lost to follow-up.

Intervention: SCIT Versus Placebo (or Other Therapy Except AIT). Primary outcome measures were (1) asthma symptom scores, (2) asthma medication scores, and (3) lung function tests $\left(\mathrm{FEV}_{1}\right.$, peak expiratory flow [PEF], percent-of-predicted FVC). Secondary outcome measures were specific antibody levels (allergen-specific immunoglobulin E [sIgE], sIgG4) and adverse events.

We searched MEDLINE, Embase, PubMed, and Chinese academic databases, including China Knowledge Resource Integrated Database (CNKI), Wanfang, and Vendor Information Pages (VIP), based on the Cochrane Collaboration guidelines from January 1990 to February 2013. We used the following key words for literature search: asthma, mite, HDM, injection, subcutaneous immunotherapy, SCIT, hyposensitize, and randomized controlled trial and the equivalent Chinese terms. We also searched for any studies in conference literature sources, but these did not yield any additional published studies.

\section{Quality Assessment and Data Extraction}

Data on the general characteristics of the studies (basic information, sample sizes, randomization process, concealment of allocation procedures, blinding, intention to treat, interventions, comparisons, outcomes) were extracted. Study quality was independently rated by 2 coauthors, and cross-checking was performed to ensure validation. When any disparity arose, the investigators met with a third author as the final adjudicator to resolve the debate. Methodological quality of the literature was assessed according to the Jadad score scale (1-3, low quality; 4-7, high quality). ${ }^{5}$ 


\section{Statistical Analysis}

Statistical analysis was performed using the ReVMan 5.0 software from the Cochrane Collaboration. A chi-square test was used to test for statistical heterogeneity between trials. A fixed-effects model was used in the meta-analysis if there was no statistical heterogeneity $\left(P>.1, \mathrm{I}^{2}<50 \%\right)$; however, when there was statistical heterogeneity $(P \leq .1$, $\mathrm{I}^{2} \geq 50 \%$ ), a random-effects model was used to reassess the data. For continuous data, the mean, SD, and sample size were combined. We used standardized mean difference (SMD) for categorical variables and relative risk as the efficacy analysis statistic for continuous variables (SCIT vs control). Confidence intervals are provided (95\% CI). $P<.05$ was considered to be statistically significant.

If data were expressed in the form of median or quartiles, the raw data were transformed to include mean and SD. The data were transformed according the Cochrane Handbook for Systematic Reviews of Interventions. ${ }^{6}$ If the outcomes were represented by histograms, graphs, or pointlike figures without any numerical data, we sent e-mails to the first author or corresponding authors to obtain the raw data.

Sensitivity analysis was performed according the Cochrane Handbook for Systematic Reviews of Interventions to evaluate the reliability and stability of the results. ${ }^{6}$ Combined effect size was analyzed after low-quality or largesample studies were excluded according to the criteria. Combined effect size was also compared with the results of prior exclusion.

\section{Results}

\section{Characteristics of Individual Studies}

A total of 923 articles (185 in Chinese and 738 in English) were found in the initial literature review from searching the electronic databases. We excluded 840 articles because they included non-RCTs or non-subcutaneous therapy. Of the remaining articles, reviews, non-clinical studies, and sublingual therapy studies were excluded. We examined the remaining 83 full-text articles, and we excluded duplicate publications or any trials with incomplete data. Ultimately, 19 studies were included in the analysis. ${ }^{7-25}$ A diagram of the search process is shown in Figure 1.

\section{Quality of Individual Studies}

The main characteristics of the 19 studies are shown in Table 1. A total of 796 subjects were included, with 452 receiving treatment and 344 serving as controls. All of the clinical trials were subjected to the baseline comparability

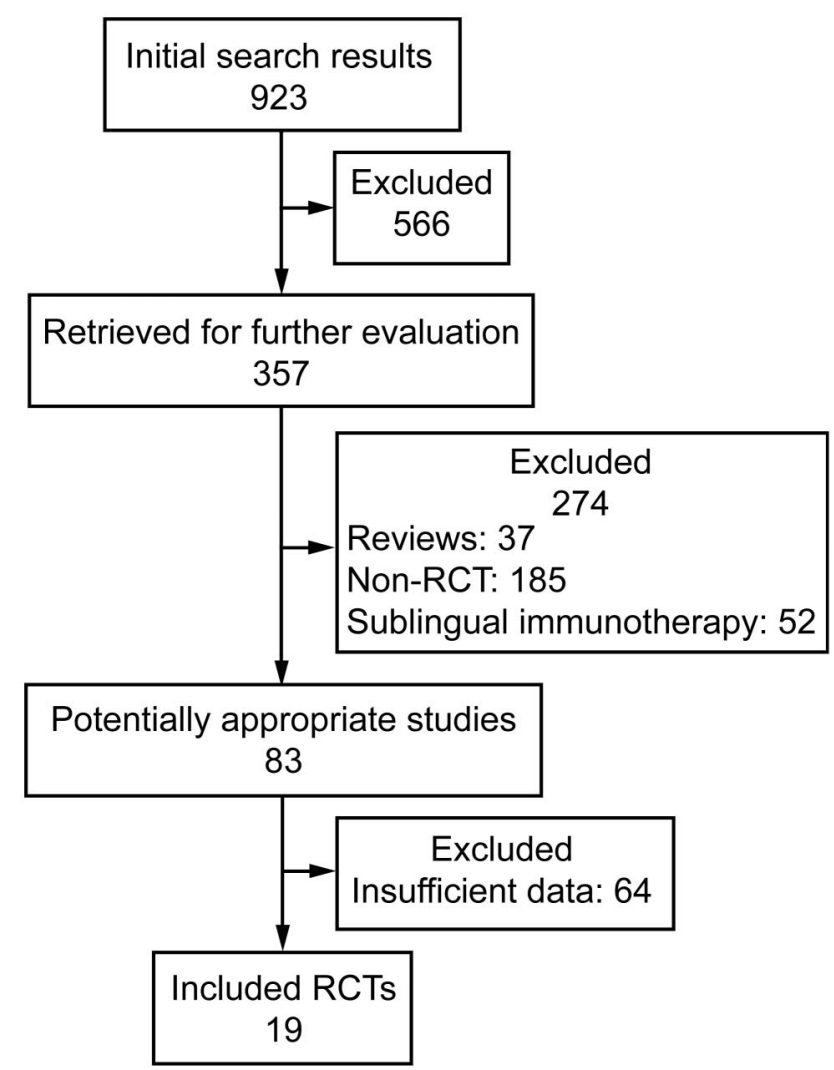

Fig. 1. Flow chart. RCT $=$ randomized controlled trial.

test, and no significant differences were found between the 2 groups with regard to age, gender, or duration and severity of disease. The methodological quality of the 19 publications was assessed using Jadad criteria (Table 2). Six articles were of high quality (four with a score of 4 and two with a score 5), and 13 articles were of low quality (two with a score of 1 , six with a score of 2 , and five with a score of 3).

\section{Results of Meta-Analyses}

Asthma Symptom Score Comparison Between SCIT and Control Groups. A total of 13 trials contained asthma symptom scores. The asthma symptom scores were extracted or converted to $\bar{x} \pm \mathrm{s}$. Data were pooled, and meta-analyses were performed using 352 SCIT subjects and 269 control subjects. The heterogeneity test was significant $(P<.001)$. A random-effects model was used after removing some heterogeneity; the SMD was -0.94 (95\% CI -1.58 to $-0.29, P=.004$ ) (Fig. 2). When the low-quality study (reference 19) was excluded, the SMD was $-1.00(95 \% \mathrm{CI}-1.69$ to $-0.31, P=.005)$. The results show that the asthma symptom scores of the SCIT group were significantly lower than those of the control group. 


\section{Subcutaneous Immunotherapy in SubJects With Asthma}

Table 1. General Characteristics of the Included Studies

\begin{tabular}{|c|c|c|c|c|}
\hline Study & Test/Control $(n)$ & Subject age (y) & Duration & Intervention and Design \\
\hline Machiels et al ${ }^{7}$ & $24 / 11$ & $16-57$ & $2 \mathrm{y}$ & $\begin{array}{l}\text { D. pteronyssinus allergen antibody complexes by } \\
2 \text { regimens/albumin buffer }\end{array}$ \\
\hline Franco et $\mathrm{al}^{8}$ & $24 / 25$ & $5-50$ & $15 \mathrm{mo}$ & Depot $D$. pteronyssinus extract/histamine placebo \\
\hline Armentia-Medina et $\mathrm{al}^{9}$ & $22 / 13$ & $13-63$ & $1 \mathrm{y}$ & Storage mite extract/open placebo \\
\hline Costa et $\mathrm{al}^{10}$ & $11 / 11$ & $12-38$ & $27 \mathrm{mo}$ & Standardized D. pteronyssinus extract/unstated \\
\hline Paranos and Petrovic ${ }^{11}$ & $7 / 7$ & $20-40$ & $6 \mathrm{mo}$ & Aqueous D. pteronyssinus extract $/ 0.9 \% \mathrm{NaCl}$ \\
\hline Pichler et al ${ }^{12}$ & $16 / 14$ & $20-46$ & $12 \mathrm{mo}$ & Depot house mite mixture (Alutard)/unstated \\
\hline Olsen et al ${ }^{13}$ & $17 / 6$ & $18-64$ & $12 \mathrm{mo}$ & D. pteronyssinus or D. farinae/histamine hydroxide \\
\hline Mungan et al ${ }^{14}$ & $10 / 11$ & $18-46$ & $1 \mathrm{y}$ & $\begin{array}{l}\text { D. pteronyssinus and D. farinae extracts/glycerol } \\
\text { saline diluent }\end{array}$ \\
\hline Altintaş et al ${ }^{15}$ & $29 / 5$ & $4-18$ & $2 \mathrm{y}$ & $\begin{array}{l}\text { Adsorbed or aqueous } D \text {. pteronyssinus extracts/open } \\
\text { placebo }\end{array}$ \\
\hline Tabar et al ${ }^{16}$ & $44 / 19$ & $5-50$ & $18 \mathrm{mo}$ & $\begin{array}{l}\text { Mite exacts by cluster or conventional } \\
\text { schedule/phenol saline }\end{array}$ \\
\hline Pifferi et al ${ }^{17}$ & $15 / 10$ & $6-14$ & $3 \mathrm{y}$ & D. pteronyssinus extracts/open placebo \\
\hline Basomba et al ${ }^{18}$ & $24 / 25$ & $14-50$ & $12 \mathrm{mo}$ & $\begin{array}{l}\text { Liposome-encapsulated } D \text {. pteronyssinus/placebo } \\
\quad \text { liposomes including solvent }\end{array}$ \\
\hline Ferrer and García-Sellés ${ }^{19}$ & $22 / 11$ & 27 & $6 \mathrm{mo}$ & $\begin{array}{l}\text { Depigmented } D \text {. pteronyssinus or } D \text {. farinae } \\
\text { extracts/untreated }\end{array}$ \\
\hline Maestrelli et al20 & $41 / 31$ & $8-43$ & $3 \mathrm{y}$ & $\begin{array}{l}\text { D. pteronyssinus or } D \text {. farinae mite extracts/histamine } \\
\text { placebo }\end{array}$ \\
\hline Tsai et $\mathrm{al}^{21}$ & $30 / 30$ & 9.37 & $1 \mathrm{y}$ & D. pteronyssinus/untreated \\
\hline García-Robaina et al22 & $27 / 27$ & 23.5 & $54 \mathrm{wk}$ & $\begin{array}{l}\text { Modified vaccines of } D \text {. pteronyssinus or } \\
\text { D. farina./placebo containing aluminum hydroxide, } \\
\text { phenol, and saline solution }\end{array}$ \\
\hline Ibero and Castillol ${ }^{23}$ & $15 / 13$ & $8-15$ & $4 \mathrm{mo}$ & $\begin{array}{l}\text { Vaccine containing depigmented polymerized extract } \\
\text { of D. pteronyssinus/open placebo }\end{array}$ \\
\hline Wang et $\mathrm{a}^{24}$ & $64 / 65$ & $6-45$ & $53 \mathrm{wk}$ & $\begin{array}{l}\text { Standardized aluminum hydroxide-absorbed } \\
\text { D. pteronyssinus/histamine dihydrochloride }\end{array}$ \\
\hline Yukselen et $\mathrm{al}^{25}$ & $10 / 10$ & 10.9 & $1 \mathrm{y}$ & $\begin{array}{l}\text { D. pteronyssinus or } D \text {. farinae mite extracts/histamine } \\
\text { dihydrochloride }\end{array}$ \\
\hline
\end{tabular}

Table 2. Quality Assessment of the Included Studies

\begin{tabular}{|c|c|c|c|c|c|}
\hline Studies & Allocation Sequence & Allocation Concealment & Blindness & Withdrawals and Dropouts & Jadad Score \\
\hline Machiels et $\mathrm{al}^{7}$ & Unclear & Unclear & Double-blind & Describe & 3 \\
\hline Franco et $\mathrm{al}^{8}$ & Unclear & Unclear & Double-blind & Describe & 3 \\
\hline Armentia-Medina et $\mathrm{al}^{9}$ & Unclear & Unclear & Double-blind & Describe & 4 \\
\hline Costa et al ${ }^{10}$ & Unclear & No & Double-blind & Describe & 3 \\
\hline Paranos and Petrovic ${ }^{11}$ & Unclear & No & Single-blind & Unknown & 1 \\
\hline Pichler et al ${ }^{12}$ & Unclear & Unclear & Double-blind & Describe & 2 \\
\hline Olsen et al ${ }^{13}$ & Yes & Yes & Double-blind & Describe & 5 \\
\hline Mungan et al ${ }^{14}$ & Unclear & Unclear & Single-blind & Describe & 2 \\
\hline Altintaş et al ${ }^{15}$ & Unclear & Unclear & Double-blind & Unknown & 2 \\
\hline Tabar et al ${ }^{16}$ & Unclear & Unclear & Unclear & Describe & 2 \\
\hline Pifferi et a $1^{17}$ & Unclear & Unclear & Single-blind & Describe & 2 \\
\hline Basomba et a ${ }^{18}$ & Unclear & Unclear & Double-blind & Describe & 3 \\
\hline Ferrer and García-Sellés ${ }^{19}$ & Unclear & No & Unclear & Describe & 1 \\
\hline Maestrelli et al ${ }^{20}$ & Unclear & Yes & Double-blind & Describe & 4 \\
\hline Tsai et al ${ }^{21}$ & Unclear & Unclear & Unclear & Describe & 2 \\
\hline García-Robaina et al22 & Unclear & Unclear & Double-blind & Describe & 4 \\
\hline Ibero and Castillo ${ }^{23}$ & Unclear & Unclear & Double-blind & Describe & 3 \\
\hline Wang et $\mathrm{al}^{24}$ & Unclear & Yes & Double-blind & Describe & 4 \\
\hline Yukselen et al 25 & Yes & Yes & Double-blind & Describe & 5 \\
\hline
\end{tabular}




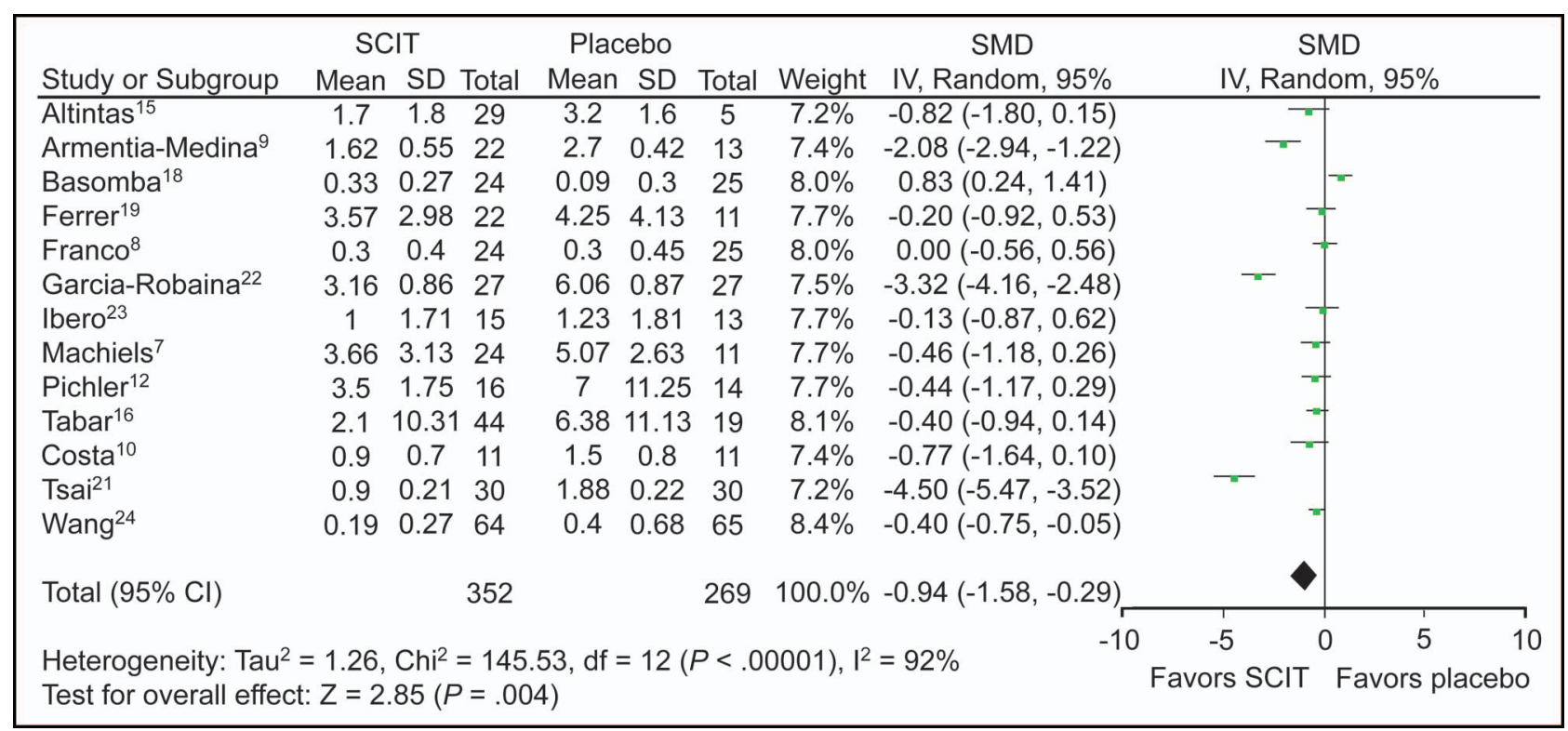

Fig. 2. Comparison of the symptom scores between the subcutaneous immunotherapy (SCIT) and control groups. SMD $=$ standardized mean difference; $\mathrm{df}=$ degrees of freedom.

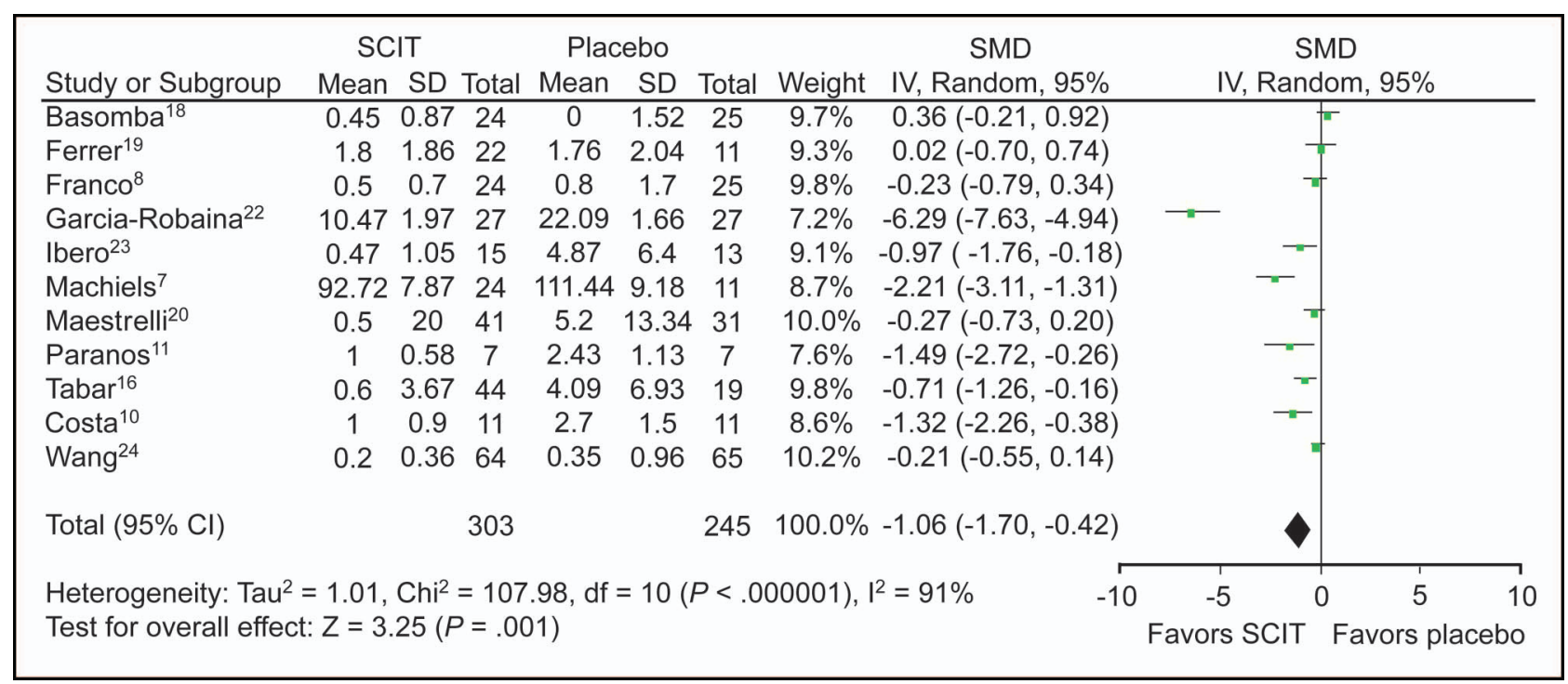

Fig. 3. Comparison of the medication scores between the subcutaneous immunotherapy (SCIT) and control groups. SMD $=$ standardized mean difference; $\mathrm{df}=$ degrees of freedom.

\section{Asthma Medication Score Comparison Between SCIT} and Control Groups. Eleven articles contained asthma medication scores. The asthma symptom scores were extracted or converted to $\bar{x} \pm \mathrm{s}$. Data were pooled, and metaanalyses were performed using 303 SCIT subjects and 245 control subjects. The heterogeneity test was significant $(P<.001)$. A random-effects model was used after removing some heterogeneity; the SMD was -1.06 (95\% CI -1.70 to $-0.42, P=.001$ ) (Fig. 3). When the low-quality study (reference 19) were excluded, the SMD was -1.18 ( $95 \% \mathrm{CI}-1.88$ to $-0.49, P=.009$ ). The results show that the asthma medication scores of the SCIT group were significantly lower than those of the control group.

Pulmonary Function Comparison Between SCIT and Control Groups. Three articles contained PEF data. The data were extracted or converted to $\bar{x} \pm \mathrm{s}$. Data were pooled, and meta-analyses were performed using 92 SCIT subjects and 55 control subjects. The results of the heterogeneity test $(P<.001)$ indicated statistical heterogeneity. A random-effects model was used after removing some heterogeneity; the SMD was -0.55 (95\% CI -1.70 to 


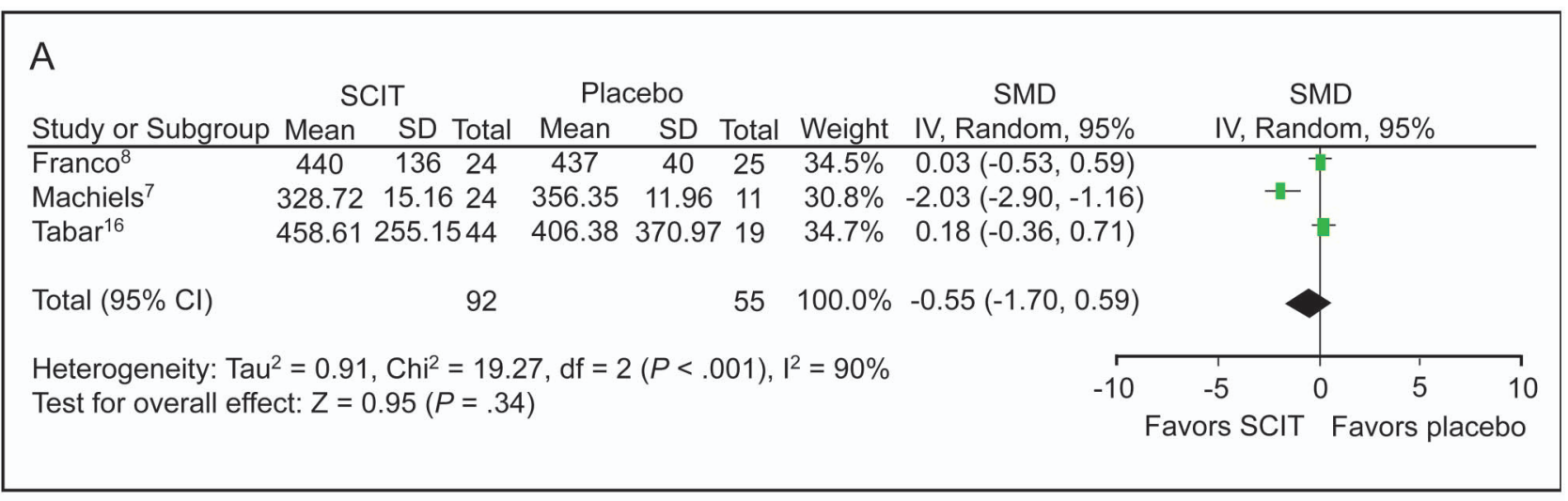

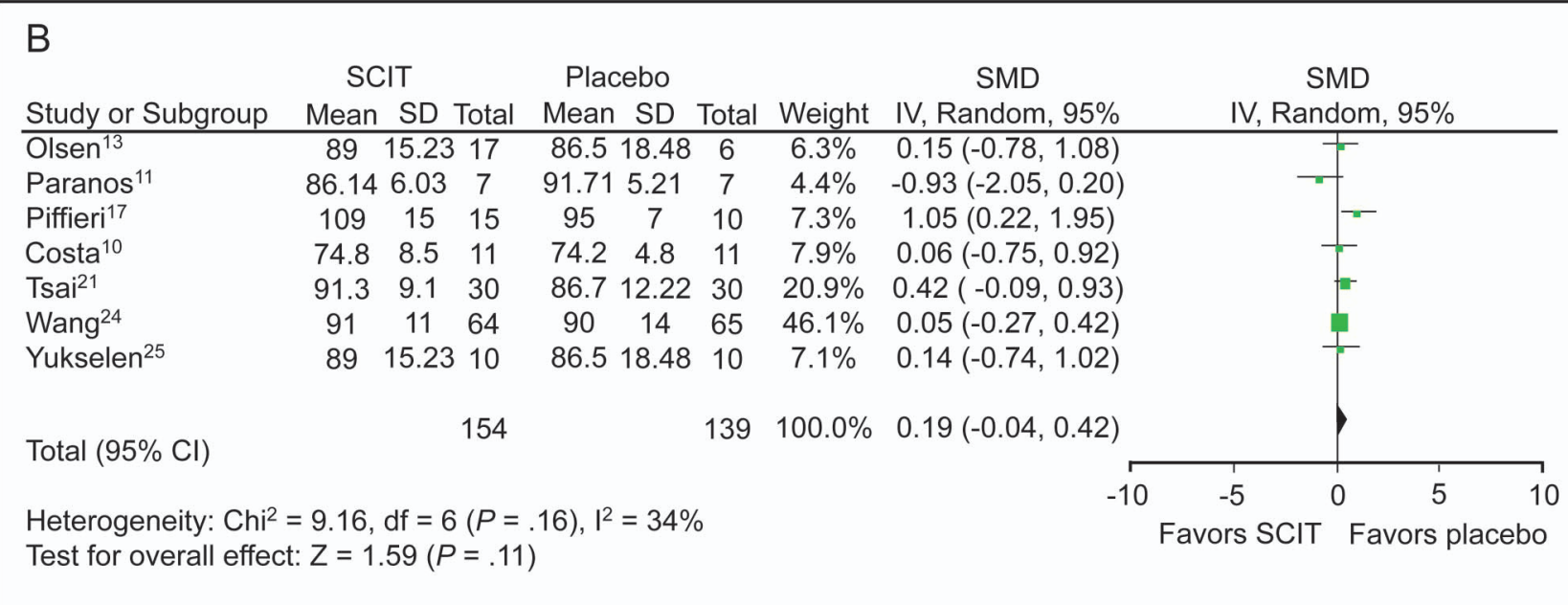

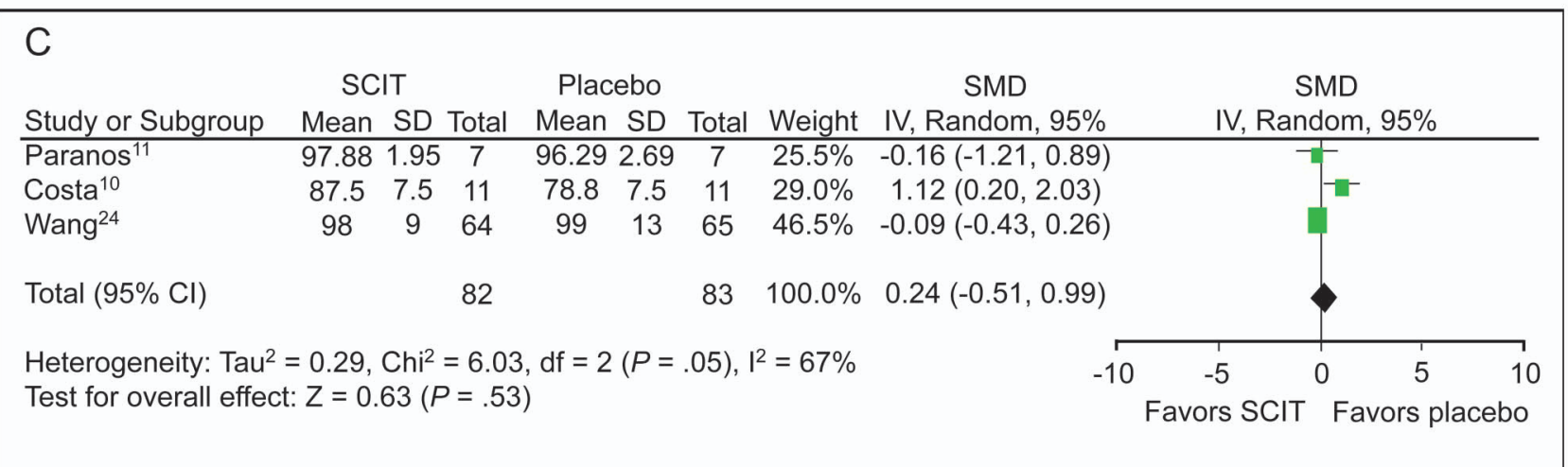

Fig. 4. Comparison of pulmonary function between the subcutaneous immunotherapy (SCIT) and control groups. A: Comparison for PEF. B: Comparison for percent-of-predicted FEV 1 . C: Comparison for percent-of-predicted FVC. SMD = standardized mean difference; $\mathrm{df}=$ degrees of freedom.

$0.59, P=.34)$ (Fig. 4A). These results showed no statistically significant difference in PEF between the SCIT and control groups. Paranos and Petrovic ${ }^{11}$ and Wang et $\mathrm{al}^{24}$ reported that subjects with and without SCIT had no difference in percent-of-predicted PEF.

Seven articles contained the percent-of-predicted $\mathrm{FEV}_{1}$. The percent-of-predicted $\mathrm{FEV}_{1}$ data were extracted or converted to $\bar{x} \pm \mathrm{s}$. Data were pooled, and meta-analyses were performed using a total of 154 SCIT subjects and 139 control subjects. With a heterogeneity test showing $P=.16$, there was statistically significant homogeneity between these studies. A fixed-effects model was used, and the SMD was 0.19 (95\% CI -0.04 to $0.42, P=.11$ ) (Fig. 4B). The results showed no statistical significance in the percent-of-predicted $\mathrm{FEV}_{1}$ between the SCIT and control groups. 


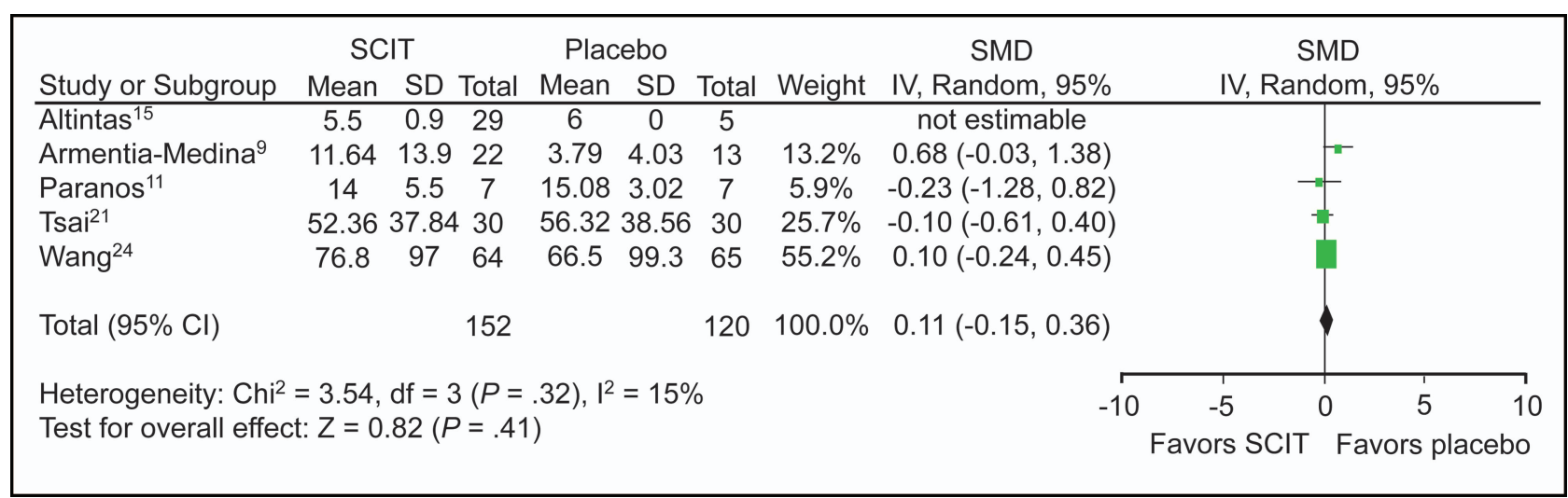

Fig. 5. Comparison of allergen-specific immunoglobulin E (slgE) between the subcutaneous immunotherapy (SCIT) and control groups. $\mathrm{SMD}=$ standardized mean difference; $\mathrm{df}=$ degrees of freedom.

Three articles contained the percent-of-predicted FVC. The percent-of-predicted FVC data were extracted or converted to $\bar{x} \pm \mathrm{s}$. Data were pooled, and meta-analyses were performed using a total of 82 SCIT subjects and 83 control subjects. The heterogeneity test showed $P=.05$, indicating statistical heterogeneity. A random-effects model was used, and the SMD was 0.24 (95\% CI -0.51 to 0.09 , $P=.53$ ) (Fig. 4C), indicating no statistical significance in the percent-of-predicted FVC between the SCIT and control groups.

\section{Serological Antibody Comparison Between SCIT and} Control Groups. Five articles referred to sIgE. The sIgE data were extracted or converted to $\bar{x} \pm \mathrm{s}$. Data were pooled, and meta-analyses were performed using a total of 152 SCIT subjects and 120 control subjects. The results of the heterogeneity test $(P=.32)$ indicated homogeneity between these studies. A fixed-effects model was used, and the SMD was 0.11 (95\% CI -0.15 to $0.36, P=.41)$ (Fig. 5), indicating no statistical significance in sIgE between the SCIT and control groups. Two articles contained data on $\operatorname{sgG} 4$, and no differences were reported between the SCIT and control groups.

\section{Adverse Event Comparison Between SCIT and Con-} trol Groups. Eight articles contained data on the safety of SCIT. No systemic reactions were reported in 3 of these studies; $9.1 \%$ of subjects (8/88) with SCIT and $2.7 \%$ of subjects $(2 / 88)$ without SCIT had systemic reactions in the other 5 studies. In addition, $17.2 \%$ of subjects with SCIT (23/134) and $8.1 \%$ of subjects (8/99) without SCIT had local reactions in 7 studies. Subjects receiving SCIT were at a higher incidence for adverse events, even though our analyses showed no statistical differences compared with subjects without SCIT: the systemic reaction pooled relative risk was 2.70 (95\% CI 0.82-8.95, $P=.10$ ), and the local reaction pooled relative risk was $1.96(95 \% \mathrm{CI}$ $0.96-3.99, P=.06$ ) (Fig. 6). All reported adverse events were mild (grade 2 or less) local reactions, including redness, itching, swelling, or hives at the injection site, usually occurring 20-30 min after injection.

\section{Discussion}

Asthma symptom and asthma medication scores are the 2 most important clinical indicators for the evaluation of AIT efficacy. Thirteen studies that met the inclusion criteria reported asthma symptom scores. Meta-analysis results showed that the symptom scores of the SCIT group were significantly lower than those of the control group, and these results suggest that SCIT could relieve clinical symptoms. A high heterogeneity between studies was noted, so we performed sensitivity analyses to produce stable results after removing low-quality literature. We did this to exclude bias related to low-quality publications. We considered that high heterogeneity was related to the fact that different standards and methods were used in these different studies. Asthma symptom scores were used to evaluate the severity of disease, so it is necessary to standardize the scoring of asthma and make it an objective record. We also analyzed medication use, which we found was significantly less in the SCIT group than in the matched control group. This suggests that SCIT can reduce the use of symptomatic drugs. We are aware that it is necessary to use a variety of symptomatic drugs, including inhaled corticosteroids and bronchodilators, to relieve symptoms in the beginning of AIT because of the time necessary for AIT to begin working. Therefore, AIT for patients with asthma should be combined with symptomatic drugs and glucocorticoids. ${ }^{26}$

We also analyzed the effect of SCIT on pulmonary function in dust mite allergic asthma. A total of 10 studies met the inclusion criteria and provided lung function results, including PEF, percent-of-predicted PEF, percent-ofpredicted $\mathrm{FEV}_{1}$, and percent-of-predicted FVC. The meta- 


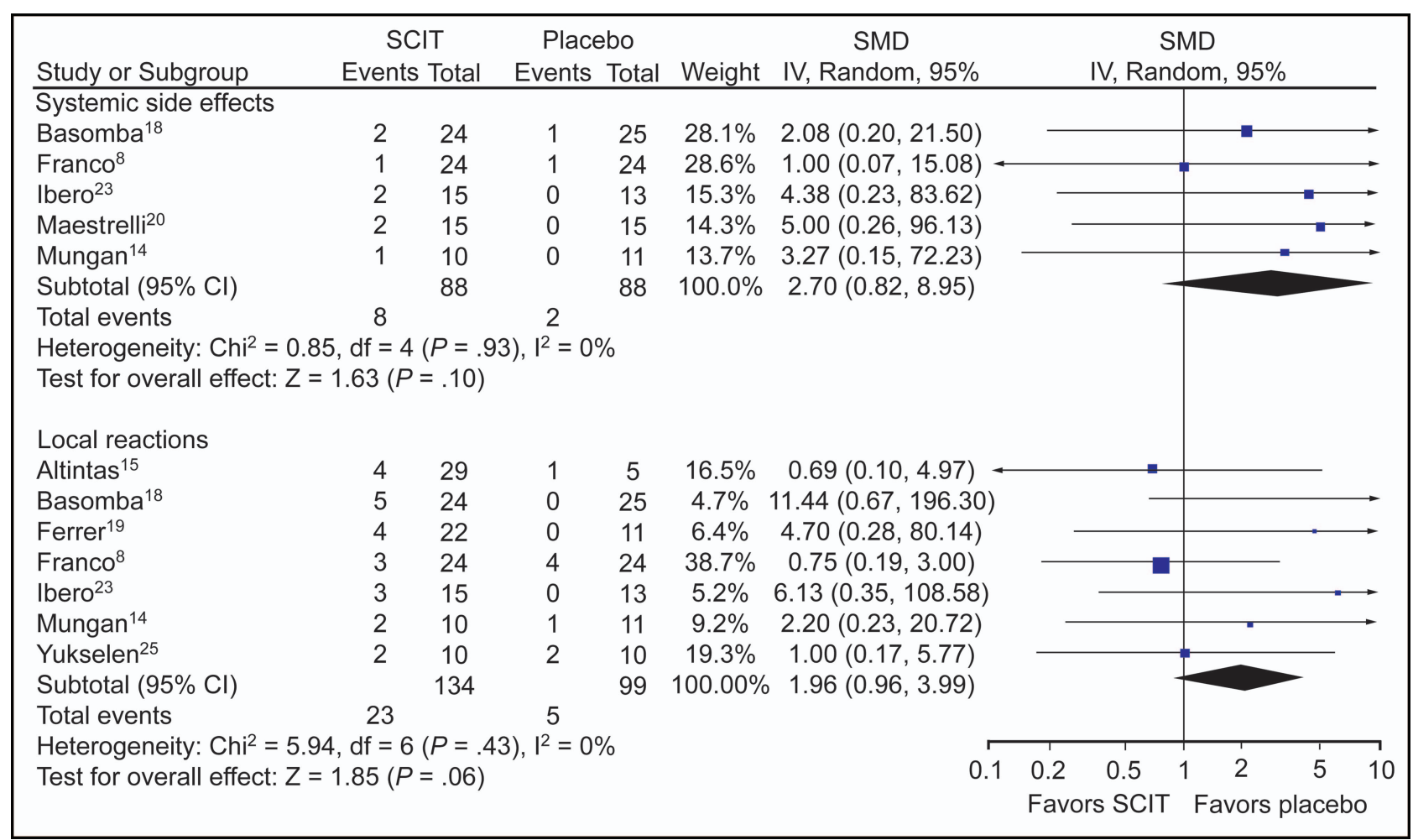

Fig. 6. Comparison of adverse events between the subcutaneous immunotherapy (SCIT) and control groups. SMD = standardized mean difference; $\mathrm{df}=$ degrees of freedom.

analysis results did not show that SCIT significantly improved lung function. This could be because most of the subjects had mild-to-moderate asthma. The studies did not include asthma exacerbations, and lung function was already restored to normal levels, so most subjects were in a non-acute phase during treatment. ${ }^{27}$

We also analyzed the changes in antigen-specific antibodies as a secondary outcome of SCIT. A study on the therapeutic mechanism of AIT described the change in sIgE and sIgG4 levels associated with host allergy and normal immune response to allergens. ${ }^{28}$ It was reported that $\operatorname{sgE}$ levels increased temporarily during the early phase of AIT but fell back to pretreatment levels during maintenance therapy. It was found that AIT also induced sIgG4 and that IgG4 antibodies acted as blocking antibodies by engaging low-affinity Fc receptors for immunoglobulin (eg, Fc $\gamma$ receptor II) expressed by basophils and mast cells, preventing an inflammatory response. Our metaanalysis results showed that there were no statistically significant differences in SIgE and sIgG4 levels between the SCIT and control groups, so the levels of sIgE and sIgG4 may be inappropriate indicators of SCIT effects. Similarly, total serum IgE levels cannot be an independent factor to reflect the atopic status of asthma, and more RCTs are needed to explore the relationship between sIgG4 and the clinical efficacy of specific immunotherapy.
The clinical effect of AIT can be categorized into 4 levels: early effect, reduction in symptoms/need for medication after finishing early-stage therapy; sustained effect, reduction in symptoms/need for medication during therapy; long-term effect, long-term reduced symptoms/need for medication after the end of the treatment period; and preventive effect, prevention of new sensitivities and progression of disease. It is difficult to evaluate the advantages and uniqueness of AIT in the early-effect and sustained-effect stages compared with other anti-asthma drugs, such as inhaled corticosteroids and leukotriene receptor antagonists. Haugaard et $\mathrm{al}^{29}$ performed a long-term controlled specific immunotherapy study using standardized extract of house dust mite antigen. Their results showed that subjects could maintain immune tolerance to dust mite antigen for $8 \mathrm{y}$ in airways, conjunctiva, and nasal mucosa. Long-term effects remained up to $5 \mathrm{y}$ after the end of therapy. Cools et al ${ }^{30}$ examined subjects with asthma who were allergic to house dust mites (D. pteronyssinus) or to both house dust mites and grass pollen and were treated with SCIT during childhood. After re-evaluating them in early adulthood after mean cessation of SCIT for $9.3 \pm 2.76 \mathrm{y}$, the results were compared with those of a control group of subjects with asthma who had comparable asthma features and were treated with appropriate anti-asthma drugs during childhood but not AIT. At 
re-evaluation, the risk of frequent asthma symptoms was 3 times higher in the control group than in the SCIT group. The frequent use of anti-asthma medication was also more pronounced in the control group. The authors concluded that SCIT has long-term effects on asthma symptoms in young adults. Inal et al ${ }^{31}$ reported a 5 -y follow-up study showing that children receiving SCIT had a lower probability of allergy compared with a control group. These results suggest that SCIT helps prevent house dust miterelated allergic diseases. Studies on the long-term effects and preventive efficacy of SCIT are rare, so more evidence is needed to support these conclusions.

The incidences of systemic and local adverse reactions were $9.1 \%$ (8/88) and $17.2 \%$ (23/134), respectively, in subjects treated with SCIT. All adverse events were mild (grade 2 or less) according to the standards of the EAACI, ${ }^{32}$ and no fatal anaphylactic shock was reported. Uncontrolled asthma was the major risk factor of AIT, so it is recommended that the status of asthma be evaluated before each allergen injection. ${ }^{33}$ Oral antihistamine also could reduce the incidence and extent of adverse reactions. ${ }^{34}$ In our pooled analyses, subjects receiving SCIT were at higher risk for adverse events, although there was no statistical significance due to low statistical power. In this adverse event analysis, all included studies had small sample sizes, and the pooled sample sizes were also small; a smaller sample size will result in lower statistical power and is more likely to make a type-2 error that fails to reject null hypothesis. It is easy to generate subcutaneous induration after injection of an aluminum hydroxide sustained-release preparation for a short time, but the indurations could dissipate themselves. We think that it is not necessary to stop therapy even though there is a relatively higher incidence of adverse events. Instead, the dose could be regulated based on the condition. In conclusion, adverse events of patients with SCIT are mild, and the safety of SCIT is acceptable.

Our meta-analysis had several limitations. First, a total of 19 articles representing 796 subjects were used, so our study had a relatively small sample size with limited statistical power. Second, there were only 6 high-quality articles remaining after using the modified Jadad scores. Thirteen articles were randomized, double-blinded, placebo-controlled trials. Three articles did not mention blinding, and 3 articles used single blinding. Most of the articles did not mention allocation concealment and did not report the use of intention-to-treat analysis. Therefore, selection bias and measurement bias may have occurred and limited the power of the evidence. Third, there were differences in the score standard of symptoms and medication in the studies, and the data were not all expressed in the same way: some were expressed in charts or only as mean and median without providing $\bar{x} \pm \mathrm{SD}$. These issues complicated the meta-analysis. Considering the limited quality of the studies included in this analysis, we suggest that further experimental work should be rigorously designed to decrease bias. Most of the studies did not have the same treatments and follow-up times and used allergen extracts from various sources. These factors may be associated with the differential clinical effects. In addition, there was significant heterogeneity among the different studies. Given the limitations above, our results should be interpreted with caution, and future studies are necessary.

\section{Conclusions}

In conclusion, our results provide evidence to support the hypothesis that SCIT can reduce the asthma symptom and asthma medication scores of patients with dust mite allergies. SCIT is safe and has less severe systemic adverse reactions. There was insufficient evidence to demonstrate that SCIT can significantly affect lung function (PEF, percent-of-predicted $\mathrm{FEV}_{1}$, percent-of-predicted FVC) or specific antibodies (sIgE, sIgG4). To obtain more reliable results, further investigation is necessary. Future studies should be designed with standard clinical measures. The clinical trials should be multi-center RCT studies using standardized allergen extract and with sufficient sample size, and the SCIT clinical effect should be examined on all 4 levels.

\section{REFERENCES}

1. Bousquet J, Khaltaev N, Cruz AA, Denburg J, Fokkens WJ, Togias A, et al. Allergic Rhinitis and Its impact on Asthma (ARIA) 2008 update (in collaboration with the World Health Organization, GA(2)LEN and AllerGen). Allergy 2008;63(Suppl 86):8-160.

2. Madonini E, Musarra A. Allergen immunotherapy in asthma: current evidence and future requirements. Eur Ann Allergy Clin Immunol 2011;43(4):103-110.

3. Adkinson NF Jr, Eggleston PA, Eney D, Goldstein EO, Schuberth $\mathrm{KC}$, Bacon JR, et al. A controlled trial of immunotherapy for asthma in allergic children. N Engl J Med 1997;336(5):324-331.

4. Wang HY, Lin XP, Hao CL, Zhang CQ, Sun BQ, Zheng JP, et al. [Effect of 1-year specific immunotherapy with standardized house dust mite vaccine on mild to moderate allergic asthmatic patients]. Zhonghua Jie He He Hu Xi Za Zhi 2006;29(10):679-687. Article in Chinese.

5. Jadad AR, Moore RA, Carroll D, Jenkinson C, Reynolds DJ, Gavaghan DJ, McQuay HJ. Assessing the quality of reports of randomized clinical trials: is blinding necessary? Control Clin Trials 1996;17(1):1-12.

6. Higgins JP, Green S, editors. Cochrane handbook for systematic reviews of interventions, version 5.1.0. Updated March 2011. The Cochrane Collaboration; 2011. www.cochrane-handbook.org. Accessed October 7, 2014.

7. Machiels JJ, Somville MA, Lebrun PM, Lebecque SJ, Jacquemin MG, Saint-Remy JM. Allergic bronchial asthma due to Dermatophagoides pteronyssinus hypersensitivity can be efficiently treated by inoculation of allergen-antibody complexes. J Clin Invest 1990; 85(4):1024-1035

8. Franco C, Barbadori S, Freshwater LL, Kordash TR. A double-blind, placebo controlled study of Alpare mite D. pteronyssinus immuno- 


\section{Subcutaneous Immunotherapy in Subjects With Asthma}

therapy in asthmatic patients. Allergol Immunopathol 1995;23(2): 58-66.

9. Armentia-Medina A, Tapias JA, Martín JF, Ventas P, Fernández A. Immunotherapy with the storage mite lepidoglyphus destructor. Allergol Immunopathol 1995;23(5):211-223.

10. Costa JC, Plácido JL, Silva JP, Delgado L, Vaz M. Effects of immunotherapy on symptoms, PEFR, spirometry, and airway responsiveness in patients with allergic asthma to house-dust mites (D. pteronyssinus) on inhaled steroid therapy. Allergy 1996;51(4):238-244.

11. Paranos S, Petrovic S. Early effects of rush immunotherapy with Dermatophagoides pteronyssinus in asthmatics. J Investig Allergol Clin Immunol 1997;7(6):588-595.

12. Pichler CE, Marquardsen A, Sparholt S, Løwenstein H, Bircher A, Bischof M, Pichler WJ. Specific immunotherapy with Dermatophagoides pteronyssinus and $D$. farinae results in decreased bronchial hyperreactivity. Allergy 1997;52(3):274-283.

13. Olsen OT, Larsen KR, Jacobsan L, Svendsen UG. A 1-year, placebocontrolled, double-blind house-dust-mite immunotherapy study in asthmatic adults. Allergy 1997;52(8):853-859.

14. Mungan D, Misirligil Z, Gürbüz L. Comparison of the efficacy of subcutaneous and sublingual immunotherapy in mite-sensitive patients with rhinitis and asthma-a placebo controlled study. Ann Allergy Asthma Immunol 1999;82(5):485-490.

15. Altintaş D, Akmanlar N, Güneşer S, Burgut R, Yilmaz M, Buğdayci $\mathrm{R}$, Aksungur P. Comparison between the use of adsorbed and aqueous immunotherapy material in Dermatophagoides pteronyssinus sensitive asthmatic children. Allergol Immunopathol 1999;27(6): 309-317.

16. Tabar AI, Muro MD, García BE, Alvarez MJ, Acero S, Rico P, Olaguíbel JM. Dermatophagoides pteronyssinus cluster immunotherapy. A controlled trial of safety and clinical efficacy. J Investig Allergol Clin Immunol 1999;9(3):155-164.

17. Pifferi M, Baldini G, Marrazzini G, Baldini M, Ragazzo V, Pietrobelli A, Boner AL. Benefits of immunotherapy with a standardized Dermatophagoides pteronyssinus extract in asthmatic children: a three-year prospective study. Allergy 2002;57(9):785-790.

18. Basomba A, Tabar AI, de Rojas DH, García BE, Alamar R, Olaguíbel JM, et al. Allergen vaccination with a liposome-encapsulated extract of Dermatophagoides pteronyssinus: a randomized, doubleblind, placebo-controlled trial in asthmatic patients. J Allergy Clin Immunol 2002;109(6):943-948.

19. Ferrer A, García-Sellés J. Significant improvement in symptoms, skin test, and specific bronchial reactivity after 6 months of treatment with a depigmented, polymerized extract of Dermatophagoides pteronyssinus and D. farinae. J Investig Allergol Clin Immunol 2003; 13(4):244-251.

20. Maestrelli P, Zanolla L, Pozzan M, Fabbri LM. Effect of specific immunotherapy added to pharmacologic treatment and allergen avoidance in asthmatic patients allergic to house dust mite. J Allergy Clin Immunol 2004;113(4):643-649.

21. Tsai YG, Chien JW, Chen WL, Shieh JJ, Lin CY. Induced apoptosis of TH2 lymphocytes in asthmatic children treated with Dermatopha- goides pteronyssinus immunotherapy. Pediatr Allergy Immunol 2005; 16(7):602-608

22. García-Robaina JC, Sánchez I, de la Torre F, Fernández-Caldas E, Casanovas M. Successful management of mite-allergic asthma with modified extracts of Dermatophagoides pteronyssinus and Dermatophagoides farinae in a double-blind, placebo-controlled study. J Allergy Clin Immunol 2006;118(5):1026-1032.

23. Ibero M, Castillo MJ. Significant improvement of specific bronchial hyperreactivity in asthmatic children after 4 months of treatment with a modified extract of Dermatophagoides pteronyssinus. J Investig Allergol Clin Immunol 2006;16(3):194-202.

24. Wang H, Lin X, Hao C, Zhang C, Sun B, Zheng J, et al. A doubleblind, placebo-controlled study of house dust mite immunotherapy in Chinese asthmatic patients. Allergy 2006;61(2):191-197.

25. Yukselen A, Kendirli SG, Yilmaz M, Altintas DU, Karakoc GB. Effect of one-year subcutaneous and sublingual immunotherapy on clinical and laboratory parameters in children with rhinitis and asthma: a randomized, placebo-controlled, double-blind, double-dummy study. Int Arch Allergy Immunol 2012;157(3):288-298.

26. Chen XX, Yu Q, Yang HK, Tian JW, Yang Sk. The effect of specific immunotherapy for asthma: a systematic review. China Pra Med 2008;3(18):57-59.

27. Hui F, Li X, Kunling S. [Dynamical changes of lung function and immunologic markers in asthmatic children receiving specific immunotherapy with standardized house dust mite extract]. Zhongguo Dang Dai Er Ke Za Zhi 2010;(9):12:715-719. Article in Chinese.

28. Taher YA, Henricks PA, van Oosterhout AJ. Allergen-specific subcutaneous immunotherapy in allergic asthma: immunologic mechanisms and improvement. Libyan J Med 2010;5.

29. Haugaard L, Dahl R, Jacobsen L. A controlled dose-response study of immunotherapy with standardized, partially purified extract of house dust mite: clinical efficacy and side effects. J Allergy Clin Immunol 1993;91(3):709-722.

30. Cools M, Van Bever HP, Weyler JJ, Stevens WJ. Long-term effects of specific immunotherapy, administered during childhood, in asthmatic patients allergic to either house-dust mite or to both house-dust mite and grass pollen. Allergy 2000;55(1):69-73.

31. Inal A, Altintas DU, Yilmaz M, Karakoc GB, Kendirli SG, Sertdemir Y. Prevention of new sensitizations by specific immunotherapy in children with rhinitis and/or asthma monosensitized to house dust mite. J Investig Allergol Clin Immunol 2007;17(2):85-91.

32. Malling HJ. The position of immunotherapy in the European Academy of Allergology and Clinical Immunology. J Investig Allergol Clin Immunol 1997;7(5):356-357.

33. Cox L, Nelson H, Lockey R, Calabria C, Chacko T, Finegold I, et al. Allergen immunotherapy: a practice parameter third update. J Allergy Clin Immunol 2011;127(1 Suppl):S1-S55.

34. Nielsen L, Johnsen CR, Mosbech H, Poulsen LK, Malling HJ. Antihistamine premedication in specific cluster immunotherapy: a double-blind, placebo-controlled study. J Allergy Clin Immunol 1996; 97(6):1207-1213. 